Original Article

\title{
Age-related changes in standing ability on a foam surface based on the center-of-mass acceleration of each body segment
}

\author{
Yahiko TAKeuchi, RPT, PhD ${ }^{1 *}$, Kimiya Fujio, RPT, PhD ${ }^{2)}$, TAKeshi InAGaki, RPT, PhD ${ }^{1)}$, \\ Ryo Fukata, RPT ${ }^{1)}$, Ryota Kuroiwa, RPT ${ }^{1)}$, Atsushi Murata, MD, PhD ${ }^{1)}$ \\ 1) Department of Rehabilitation, Chiba University Hospital: 1-8-1 Inohana, Chuoku, Chiba 260-8677, \\ Japan \\ 2) Department of Rehabilitation for the Movement Functions, Research Institute of the National Reha- \\ bilitation, Center for Persons with Disabilities, Japan
}

\begin{abstract}
Purpose] The purpose of this study was to elucidate the age-related changes in the stability of the quiet standing posture based on the acceleration of the center of mass of each body segment under deteriorated somatosensory conditions. [Participants and Methods] The participants in this study were 18 healthy elderly persons and 11 healthy young adults. A foam surface was placed on the force plate for load-bearing onto the somatosensory system. The participants maintained a quiet position on the force plate under two conditions: a firm surface and a foam surface. The accelerations of the head, thorax, pelvis, and whole body center of mass when quiet standing in two conditions were measured by a motion capture system. In the statistical analysis, regarding the center of mass of each body segment, the interactions were examined by performing a two-way analysis of variance using age and surface condition as factors. [Results] A two-way analysis of variance detected an interaction between age and surface factors for anteroposterior acceleration at the center of mass of the head. For other body segments, interactions between the two factors were not detected. [Conclusion] The results of anteroposterior acceleration at the center of mass of the head suggest that under conditions of deteriorated somatosensory function in the lower limbs, minute anteroposterior position adjustment of the head is an essential characteristic of the standing posture control mechanism in the elderly.

Key words: Elderly, Acceleration of center of mass, Quiet standing
\end{abstract}

(This article was submitted Apr. 21, 2020, and was accepted Jun. 3, 2020)

\section{INTRODUCTION}

The human body has a complex segmental structure comprising the head, the trunk, and the four limbs. In a standing posture, the lines from the center of mass of each body segment to the floor do not coincide ${ }^{1)}$. Because of this, even when standing still, the body sways minutely. This body sway is the control mechanism for maintaining human standing posture ${ }^{2)}$. In other words, it is thought that by shifting each of the body segments, the sway provides a control mechanism in which the projection axis from the center of mass in space is maintained within the basal support plane of both feet. Moreover, due to age-related changes in the spinal column support mechanism in the elderly, changes in posture alignment, such as posterior pelvic tilt to compensate for the rear curvature of the entire spinal column, mainly in the thoracic part, often occur ${ }^{3)}$. Because of this, in addition to evaluation of the sway at the center of mass of the whole body, it is also necessary to understand the characteristics of the sway at the center of mass of each of the body segments.

In addition to the above mechanism, input information from various systems such as visual, somatosensory, and vestibular

*Corresponding author. Yahiko Takeuchi (E-mail: yahiko.takeuchi@chiba-u.jp)

(C2020 The Society of Physical Therapy Science. Published by IPEC Inc.

(c) (1) $\odot$ This is an open-access article distributed under the terms of the Creative Commons Attribution Non-Commercial No DerivaCC BY NC ND tives (by-nc-nd) License. (CC-BY-NC-ND 4.0: https://creativecommons.org/licenses/by-nc-nd/4.0/) 
systems is also critical in standing posture control of the human body ${ }^{4}$. In particular, somatosensory information from the lower limbs is very important for standing posture control ${ }^{5)}$.

The purpose of this study was to elucidate the age-related changes in the stability of the quiet standing posture based on the acceleration of the center of mass of each body segment under deteriorated somatosensory conditions.

\section{PARTICIPANTS AND METHODS}

The participants in this study were 18 healthy elderly persons ( 9 females, 9 males: average age $67.1 \pm 3.4$ years) and 11 healthy young adults ( 4 females, 7 males: average age $20.5 \pm 1.4$ years).

A motion capture system (Motion Analysis Co., Mac3D) comprising eight infrared cameras was used for measuring the center of mass in the quiet standing posture.

Using the Helen Hays marker set as a reference, infrared reflective markers were affixed at a total of 25 target points on each participant's body. In addition, the center of foot pressure, which is the projection point of the center of mass on the floor, was measured using force plates (AMTI Inc., BP400600). For the data sampling frequency, the camera was set at $100 \mathrm{~Hz}$, the force plate was set at $1,000 \mathrm{~Hz}$, and they were synchronized using the onboard computation of a personal computer.

The participants stood barefoot on the force plate, maintaining a quiet standing posture with a gap of $150 \mathrm{~mm}$ between the inner edges of both feet. They were asked to hold their gaze upon a target placed at a distance of $3 \mathrm{~m}$ in front of them. The measurement time for the quiet standing posture was set at $30 \mathrm{~s}$, and the data for analysis was gathered by excluding the data from the first $10 \mathrm{~s}$ after the start of the measurement.

A foam surface (AIREX Inc., Balance-pad Plus, $400 \mathrm{~mm} \times 300 \mathrm{~mm} \times 65 \mathrm{~mm}$ ) was placed on the force plate for loadbearing onto the somatosensory system. The Balance-pad Plus is manufactured using the soft foam and widely used to improve balance in physical therapy and rehabilitation and in sports exercises ${ }^{6}$. Maintaining the quiet standing posture on the force plate was termed the firm surface condition and maintaining the quiet standing posture on the Balance-pad Plus was termed the foam surface condition.

Motion analysis software (C-motion Inc., Visual3D) was used for data analysis of the center of mass and the center of foot pressure for the quiet standing posture. Based on the gauge data obtained from the motion capture system, a segmental model of the whole body consisting of the head, thorax, and pelvis was developed to calculate the center of mass of each body segment. Regarding the inertia coefficient for each body segment model, the values reported by Dempster ${ }^{7)}$ were used as a reference, and the method proposed by Winter ${ }^{1)}$ was used for calculating the center of mass. In addition, for $20 \mathrm{~s}$ during the quiet standing posture, the center of mass of each body segment and the effective value of acceleration in the anteriorposterior, and lateral directions at the center of foot pressure were calculated.

In the statistical analysis, regarding the center of mass of each body segment and the acceleration value at the center of foot pressure, the interactions were examined by performing a two-way analysis of variance using age (elderly, young) and surface condition (firm, foam) as factors. The significance level was set at $5 \%$.

All participants received verbal and written explanations of the purpose of the experiment and received written consent. This study was approved by the ethics committee of the Chiba Prefectural University of Health Sciences, which is the former institution of the first author.

\section{RESULTS}

Table 1 shows the data for the center of mass of each body segment and the acceleration value at the center of foot pressure under firm and foam conditions respectively. As a result of two-way analysis of variance, the interaction between the age factor and the surface factor was detected in the anteroposterior acceleration values of the center of mass of the head $(F=19.7$,

Table 1. Acceleration at the center of mass of each body segment and at the center of foot pressure under different surface conditions

\begin{tabular}{|c|c|c|c|c|c|c|c|c|c|c|}
\hline & \multicolumn{10}{|c|}{ Elder } \\
\hline & \multicolumn{2}{|c|}{ Head } & \multicolumn{2}{|c|}{ Thorax } & \multicolumn{2}{|c|}{ Pelvis } & \multicolumn{2}{|c|}{ Whole body } & \multicolumn{2}{|c|}{$\mathrm{COP}$} \\
\hline & AP & ML & AP & ML & $\mathrm{AP}$ & ML & AP & ML & AP & ML \\
\hline Firm & $0.10 \pm 0.03$ & $0.07 \pm 0.02$ & $0.19 \pm 0.06$ & $0.05 \pm 0.01$ & $0.10 \pm 0.06$ & $0.04 \pm 0.02$ & $0.08 \pm 0.02$ & $0.03 \pm 0.01$ & $0.23 \pm 0.07$ & $0.14 \pm 0.03$ \\
\hline \multirow[t]{4}{*}{ Foam } & $0.17 \pm 0.05$ & $0.11 \pm 0.02$ & $0.33 \pm 0.07$ & $0.09 \pm 0.02$ & $0.17 \pm 0.03$ & $0.08 \pm 0.01$ & $0.13 \pm 0.03$ & $0.04 \pm 0.01$ & $0.45 \pm 0.16$ & $0.25 \pm 0.10$ \\
\hline & \multicolumn{10}{|c|}{$\begin{array}{ll} & \text { Young } \\
\end{array}$} \\
\hline & \multicolumn{2}{|c|}{ Head } & \multicolumn{2}{|c|}{ Thorax } & \multicolumn{2}{|c|}{ Pelvis } & \multicolumn{2}{|c|}{ Whole body } & \multicolumn{2}{|c|}{ COP } \\
\hline & AP & ML & $\mathrm{AP}$ & ML & AP & ML & $\mathrm{AP}$ & ML & AP & ML \\
\hline Firm & $0.08 \pm 0.01$ & $0.06 \pm 0.01$ & $0.16 \pm 0.03$ & $0.05 \pm 0.01$ & $0.08 \pm 0.01$ & $0.03 \pm 0.01$ & $0.06 \pm 0.01$ & $0.02 \pm 0.00$ & $0.19 \pm 0.03$ & $0.13 \pm 0.02$ \\
\hline Foam & $0.11 \pm 0.02$ & $0.09 \pm 0.02$ & $0.26 \pm 0.07$ & $0.07 \pm 0.02$ & $0.14 \pm 0.03$ & $0.06 \pm 0.01$ & $0.11 \pm 0.03$ & $0.04 \pm 0.01$ & $0.29 \pm 0.12$ & $0.21 \pm 0.07$ \\
\hline
\end{tabular}

Mean $\pm \mathrm{SD}, \mathrm{m} / \mathrm{s}^{2}$.

COP: Center of pressure; AP: Anteroposterior; ML: Mediolateral; Firm: Firm surface; Foam: Foam surface. 


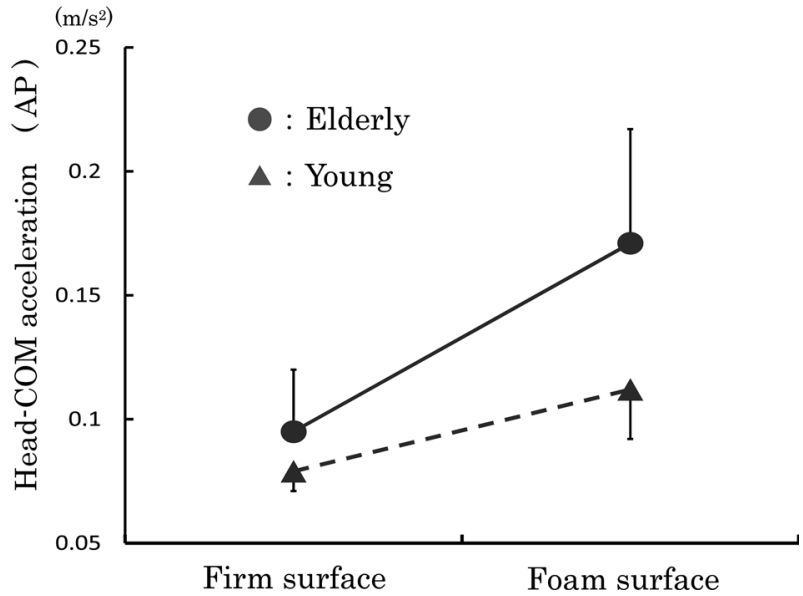

Fig. 1. Comparison of anteroposterior acceleration at the center of mass of the head. COM: Center of mass; AP: anteroposterior

$\mathrm{p}<0.01)$ and the center of foot pressure $(\mathrm{F}=11.6, \mathrm{p}<0.01)$. Figure 1 shows the interaction diagram between the age factor and the surface factor in the anteroposterior acceleration at the center of mass of the head.

For acceleration at the centers of mass of other body segments, interactions between the two factors were not detected.

\section{DISCUSSION}

In previous studies, quiet standing on a foam surface reduces the reliability of somatosensory information and increases postural sway ${ }^{6,8,9)}$.

The results of this study suggest interactions between the age and surface factors in terms of the effect on anteroposterior acceleration at the center of mass of the head. This suggests that the increase in anteroposterior sway of the head is due to aging and somatosensory confusion in the lower limbs. In a two-legged stable stance using a fairly stable base of support, it has been suggested that proprioceptive cues are more important than the visual and vestibular information in maintaining stability ${ }^{10)}$. In the control mechanism for maintaining a quiet standing posture, it is thought that age-related deterioration in somatosensory function from the lower limbs and, in particular, the deficiency in afferent input from the plantar mechanoreceptors, causes the increase in anteroposterior sway of the head. Within the body axis in the skeleton, the head and neck segment is where the largest three-dimensional motion is possible, and it plays an important role in optimizing the spatial positioning in sensory systems such as the visual and vestibular systems, which are critical in standing posture control ${ }^{11)}$. The results of anteroposterior acceleration at the center of mass of the head suggest that under conditions of deteriorated somatosensory function in the lower limbs, minute anteroposterior position adjustment of the head is an essential characteristic of the standing posture control mechanism in the elderly.

The results of the study also suggest that, regarding the effect on the anteroposterior acceleration at the center of foot pressure controlling the center of mass of the whole body based on the floor reaction force, interactions between the age and surface factors are present. These results suggest that in the elderly group, the anteroposterior acceleration at the center of foot pressure increases as a means to control the anteroposterior sway of the whole body, which results from a combination of the effect of aging and deterioration of somatosensory function in the lower limbs. The increase in the center of foot pressure acceleration as a means to control the center of mass is being applied in the standing posture control mechanism predictively ${ }^{12,13}$. Based on this, the study suggests that there is a need to mobilize better predictive control mechanisms to assist in maintaining a quiet standing posture in the elderly under conditions of deteriorated somatosensory function.

\section{Funding}

This work was supported by JSPS KAKENHI Grant Number 17K07594.

\section{Conflict of interest}

There are no conflicts of interest to declare. 


\section{REFERENCES}

1) Winter DA: Biomechanics and motor control of human movement, 4th ed. New York: John Wiley \& Sons, 2009, pp 82-106

2) McCollum G, Leen TK: Form and exploration of mechanical stability limits in erect stance. J Mot Behav, 1989, 21: 225-244. [Medline] [CrossRef]

3) Elble RJ: Change in gait with normal aging. In: Ma sdeu JC, et al. (eds.): Gait disorders of aging, 1st ed. Philadelphia: Lippincott-Raven, 1997 , pp 93-105.

4) Woollacott MH, Shumway-Cook A, Nashner LM: Aging and posture control: changes in sensory organization and muscular coordination. Int J Aging Hum Dev, 1986, 23: 97-114. [Medline] [CrossRef]

5) Shumway-Cook A, Horak FB: Assessing the influence of sensory interaction of balance. Suggestion from the field. Phys Ther, 1986, 66: 1548-1550. [Medline] [CrossRef]

6) Tanaka H, Uetake T: Characteristics of postural sway in older adults standing on a soft surface. J Hum Ergol (Tokyo), 2005, 34: 35-40. [Medline]

7) Dempster WT: Space requirements of the seated operator, WADC Technical Report, Wright Patterson Air Force Base,1955, pp 55-159.

8) Vuillerme N, Pinsault N: Re-weighting of somatosensory inputs from the foot and the ankle for controlling posture during quiet standing following trunk extensor muscles fatigue. Exp Brain Res, 2007, 183: 323-327. [Medline] [CrossRef]

9) Patel M, Fransson PA, Johansson R, et al.: Foam posturography: standing on foam is not equivalent to standing with decreased rapidly adapting mechanoreceptive sensation. Exp Brain Res, 2011, 208: 519-527. [Medline] [CrossRef]

10) Koceja DM, Allway D, Earles DR: Age differences in postural sway during volitional head movement. Arch Phys Med Rehabil, 1999, 80: 1537-1541. [Medline] [CrossRef]

11) Neumann DA: Kinesiology of the musculoskeletal system, 2nd ed. St. Louis: Mosby, 2010, pp 379-422.

12) Masani K, Vette AH, Kouzaki M, et al.: Larger center of pressure minus center of gravity in the elderly induces larger body acceleration during quiet standing. Neurosci Lett, 2007, 422: 202-206. [Medline] [CrossRef]

13) Yu E, Abe M, Masani K, et al.: Evaluation of postural control in quiet standing using center of mass acceleration: comparison among the young, the elderly, and people with stroke. Arch Phys Med Rehabil, 2008, 89: 1133-1139. [Medline] [CrossRef] 